\title{
Breast cancer in young women
}

\section{Part IV: Breast cancer and pregnancy}

\author{
Agustí Barnadas · Carlos Vázquez
}

Received: 1 July 2010/Accepted: 6 July 2010/Published online: 14 August 2010

(C) Springer Science+Business Media, LLC. 2010

\begin{abstract}
The proportion of women who are of childbearing age or pregnant seems to be increasing, probably due to delayed age at first pregnancy. Both clinical situations require special attention and participation of a multidisciplinary team. Breast cancer during pregnancy calls for strict monitoring of the mother and fetus. Selection of diagnostic methods, treatment and follow-up assessments should involve minimal risk to the fetus. Therefore, some diagnostic methods, such as magnetic resonance, and several treatments, such as radiotherapy and chemotherapy during the first trimester, must be avoided unless their use is strictly necessary. Therapeutic abortion is rarely indicated, except in women with metastatic disease or when chemotherapy administration is
\end{abstract}

not possible. After breast cancer, it is prudent to defer pregnancy until two years after completion of therapy due to the high risk of recurrence in this period. One of the major adverse events of cancer therapy is chemotherapyinduced ovarian failure and amenorrhea. Most chemotherapeutics increase the risk of early menopause and loss of fertility. Therefore, in young women the use of agents with the least impact on ovarian function must be considered. In addition, prior to treatment physicians should inform their patients about their reproductive future and available options to preserve fertility.

Conflict of Interest The authors have no conflict of interest.

\footnotetext{
A. Barnadas $(\square)$

Hospital Santa Creu i Sant Pau, Barcelona, Spain

e-mail: abarnadasm@santpau.cat

C. Vázquez

Instituto Valenciano de Oncología, Valencia, Spain

e-mail: cvazquez@fivo.org
} 